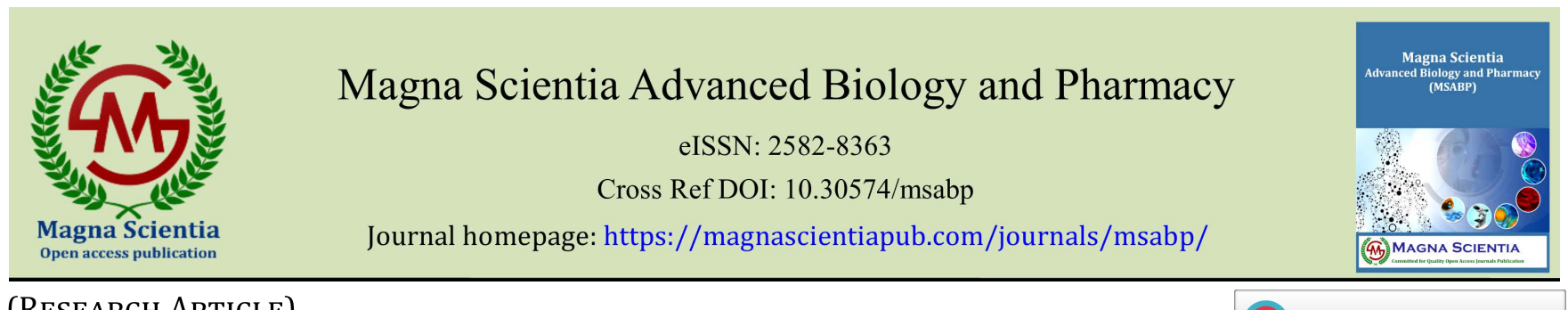

(RESEARCH ARTiClE)

Check for updates

\title{
Effect of organic mulch and LEDs light colors on green bean plants (Phaseolus vulgaris L.)
}

\author{
Fatma S Moursy * \\ Climate Modification Research Department, Central Laboratory for Agricultural Climate (CLAC), Agricultural Research \\ Center (ARC), Giza, Egypt.
}

Magna Scientia Advanced Biology and Pharmacy, 2021, 03(01), 027-044

Publication history: Received on 18 June 2021; revised on 30 July 2021; accepted on 02 August 2021

Article DOI: https://doi.org/10.30574/msabp.2021.3.1.0032

\begin{abstract}
Studying the positive role of applying organic mulch and different colors of LEDs light, on the growth and yield of green bean plants, were carried out during 2019/2020 and 2020/2021 seasons, at a privet farm within Suez Governorate. Seedlings of Bronco cultivar were transplanted at the $15^{\text {th }}$ of October of both seasons. Two types of organic mulch i.e., chopped maize straw and chopped rice straw, addition to bare soil (control) were tested as a soil mulch treatment (factor 1). In addition, LEDs light colors i.e., red, blue and "red + blue" and without LED light were also tested (factor 2). Finally, interaction between both studied factors was also studied. Studied factors were arranged in split plot design with three replicates. Organic soil mulches were arranged in the main plots. However, LEDs light colors were arranged within the sub main plots. Vegetative growth parameters i.e., plant height, number of leaves/plant, stem diameter, number of branches/plant, fresh and dry weight of plants were measured. Moreover, percentages of nitrogen, phosphorus and potassium contents in leaves, as well as, chlorophyll reading (SPAD) were also measured. In addition, pod characteristics i.e., number of pods/plant, average pod weight and fiber content in pods, were studied as well. Finally, both early and total yield of green bean were studied. Changes in soil temperature under organic mulch treatments, as well as, bare soil were daily measured during the growing seasons. Results indicated that, in general, applying tested types of organic mulch modified soil temperature. Moreover, both tested factors were enhanced measured characters of vegetative growth, leaves content of nitrogen, phosphorus and potassium, as well as yield and its components. Furthermore, both treatments of chopped maize straw and "red + blue" LEDs light, as well as the interaction between both treatments were the most suitable for increasing productivity and quality of green bean plants.
\end{abstract}

Keywords: Organic mulch; LEDs light colors; Green bean; Soil temperature; Total yield

\section{Introduction}

Green beans (Phaseolus vulgaris L.) is important vegetable crop belongs to legumes, with high protein contents, fiber, phosphorus, iron, vitamin $B_{1}$, high concentrations of essential nutrients, such as ascorbic acid, Vitamin A, Vitamin B and calcium [1]. It regulates serum cholesterol and lipid profiles, and inhibits precancerous growths [2]. Furthermore, increasing yield of green beans with quality characteristics is vital in increasing the consumption and export value of the crop. There is a need to evaluate more modern technologies that can help to meet markets and consumers requirements [3].

Covering agricultural bare soil is a practice to enhance soil properties, plant growth and crop yield [4]. Soil mulching strongly connected with different elements and factors that are used in vegetable crop intensive production systems and increase its efficiency. Many materials are valid to be used as soil mulch in respect to its impact on the characteristics

\footnotetext{
${ }^{*}$ Corresponding author: Fatma S Moursy

Climate Modification Research Department, Central Laboratory for Agricultural Climate (CLAC), Agricultural Research Center (ARC), Giza, Egypt.
} 
of soil and plant growth [5]. Furthermore, organic mulch application is a good energy source for the microorganisms in the root zone. In addition, it plays an important role in water penetration [5]. Organic mulch decomposition promotes soil nutrients through slow release from organic mulch, as well as, nourishing the agricultural soil gradually during the season [6]. From the climatological point of view, organic mulch, such as rice straw mulch, performs like an air conditioner for the soil, whereas, it makes soil temperature higher during the colder seasons and lower during the warmer seasons when compared with bare soil [7]. Temperature extremes affect plants in early stage of growth negatively through stopping nutrient uptake by the new developed roots [8]. So, adjustment and maintaining soil temperature to suit the cultivated crop is vital for avoiding such stressful conditions.

Straws from any crop are intensively available locally after harvesting. Recently, rice straw is used as soil mulch. It provides all advancement of polyethylene mulch. In addition, it presents better conservation of water through the early stage of crop growth [9]. Abd El-Wahed et al., [10] reported a significant enhancement in green bean yields (by 11.5, 30.8 and $40.2 \%$ ) as a direct result of using rice straw mulch of the following thickness 3,6 and $9 \mathrm{~cm}$, respectively, compared to bare soil.

On the other hand, light is very important factor that control plants performance. Plants showed different response to light according to the type of photo-sensory receptors. Reaction of plants to light are depending on light quality (color or wavelength), light quantity (fluency rate) and finally light direction and duration [11]. Wavelengths, affecting plant growth and development, are in the range between 300-700 nm, and called Photosynthetically active radiation (PAR) [12]. Moreover, inside the light spectrum both blue and red regions are basically producing the quantum yield of photosynthesis [13]. Red wavelength (600-700 $\mathrm{nm}$ ) and blue wavelength (420-450 $\mathrm{nm}$ ) are considered as the most effective regions of light for controlling plant growth and yield [14]. The mix between red and blue LEDs give a higher result related to accumulation of chlorophyll, antioxidants and carotenoids [15, 16, 17 and 18].

Light-Emitting Diodes (LEDs) work as an innovative tool for achieving desired performance of horticulture crops throughout fine tuning of the light conditions for cultivated plants [19]. In addition, LED light represents advancement in relation to higher efficiency in converting energy into photosynthetically active radiation, lower cooling requirements, robustness and cost effectiveness as compared to other source of light that usually used in horticulture production [20]. To adjust a specific characteristic of horticulture production, LED customized the distribution of a specific wavelength that is able to govern growth, development or metabolic processes [12].

The aim of this study is to determine the positive role of two types of organic mulches (maize straw \& rice straw) and three different wavelengths (Red, Blue and "Red + Blue) on enhancing the growth and productivity of green beans.

\section{Material and methods}

\subsection{Experimental layout}

Experiments were conducted at a privet farm in Suez Governorate, during 2019 and 2020 seasons. Seedlings of Bronco green bean cultivar were transplanted at $15^{\text {th }}$ of October in hills $(30 \mathrm{~cm}$ apart) on ridges (each is $50 \mathrm{~cm}$ width). Plot area was $30 \mathrm{~m}^{2}(30 \mathrm{~m} \times 1 \mathrm{~m})$. A distance of $150 \mathrm{~cm}$ was left between each treatment. Treatments of two types of organic mulch, three LED light colors, and their interaction, were carried out, compared with natural open field conditions, to investigate the growth and productivity of green bean plants.

\subsection{Soil preparation}

Recommended amount of fertilizers ( $17 \mathrm{Kg}$ of calcium super phosphate, $4.5 \mathrm{Kg}$ of ammonium sulphate, $8.5 \mathrm{Kg}$ of Sulphur, $4.5 \mathrm{Kg}$ of potassium sulphate and $2 \mathrm{~m}^{3}$ of compost) were mixed perfectly to create a starter. Such mix was divided into eight equal quantities; each was added to each treatment two weeks before transplanting [21]. The drip irrigation system was used.

\subsection{Organic mulch preparation}

Using a cutting machine, the two tested organic mulches were shredded to 2-4 inches. Soil beds were covered by the two types of organic mulches (chopped maize straw and chopped rice straw), by spreading them manually on the soil surface (forming $3 \mathrm{~cm}$ thickness above bar soil) after seedlings transplanting. 


\subsection{LEDs light colors preparation}

Three LEDs light colors were used to light up tested plants i.e., Red (660 nm), Blue (440 nm) and "Red + Blue". The LED light was provided by LED inter-lighting lamps, and installed $0.60 \mathrm{~m}$ above tested plants. LED light was applied to plants daily from 6 pm to $5 \mathrm{am}$.

\subsection{Treatments}

Two tested factors were applied, and their interaction as treatments, as follows:

- Organic mulch factor

Two types of organic mulch were tested i.e., (chopped maize straw and chopped rice straw), compared with natural open field conditions as control treatment.

- LED light color factor

Three LEDs light colors were used i.e., (Red, Blue and "Red + Blue", compared with natural open field conditions as control treatment.

\subsection{Climatic conditions}

Soil temperature: Maximum and minimum soil temperatures were daily recorded under organic mulch treatments using digital thermometer. The results were calculated as an average of every 10 days.

\subsection{Recorded Data}

A sample of 3 randomized green bean plants, from each replicate in different treatments, were collected after 70 days from transplanting, to determine the following parameters i.e., plant length, number of leaves per plant, stem diameter, number of branches/plant and fresh and dry weights of total plant. In addition, number of pods per plant and average pod weight for each treatment were estimated from the first harvest to the end of each season, after that, the early and total yield per plant were estimated.

The percentage of $\mathrm{N}, \mathrm{P}$ and $\mathrm{K}$ contents were determined in leave samples after drying at $70{ }^{\circ} \mathrm{C}$ in an air forced oven for 24 hours. Dried leaves were digested in $\mathrm{H}_{2} \mathrm{SO}_{4}$, the following mineral contents were estimated: phosphorous and potassium in the acid digested solution by colorimetric method, ammonium molybdate by spectrophotometer and flame photometer [22]. Total nitrogen was determined by Kjeldahl method according to the procedure described by [23]. The total fibre content was also estimated, in green pods on dry weight basis, according to [24].

\subsection{Experimental design}

The experiment was split plot design with three replicates. The organic mulch type treatments were arranged as the main plots, whereas, the LED light colors were arranged as the sub plots.

\subsection{Data analysis}

Obtained data were statistically analyzed using the analysis of variance method. Duncan's multiple range tests at $5 \%$ level of probability were used to compare treatment means [25].

\section{Results}

\subsection{Soil temperature}

Data in Figure (1) showed the positive effect of organic mulch application on increasing average max. \& min. soil temperatures under both tested types of organic mulch. Average maximum and minimum soil temperatures were increased compared to bare soil during 2019 and 2020 seasons. Soil temperature under two tested organic mulch starts increased gradually after 20 days from applying the organic mulch in both studied seasons. 
Average max. \& min. soil temperatures $\left(c^{\circ}\right)$ of bare soil and under two types of organic mulch (rice straw \& maize straw) during season of 2019

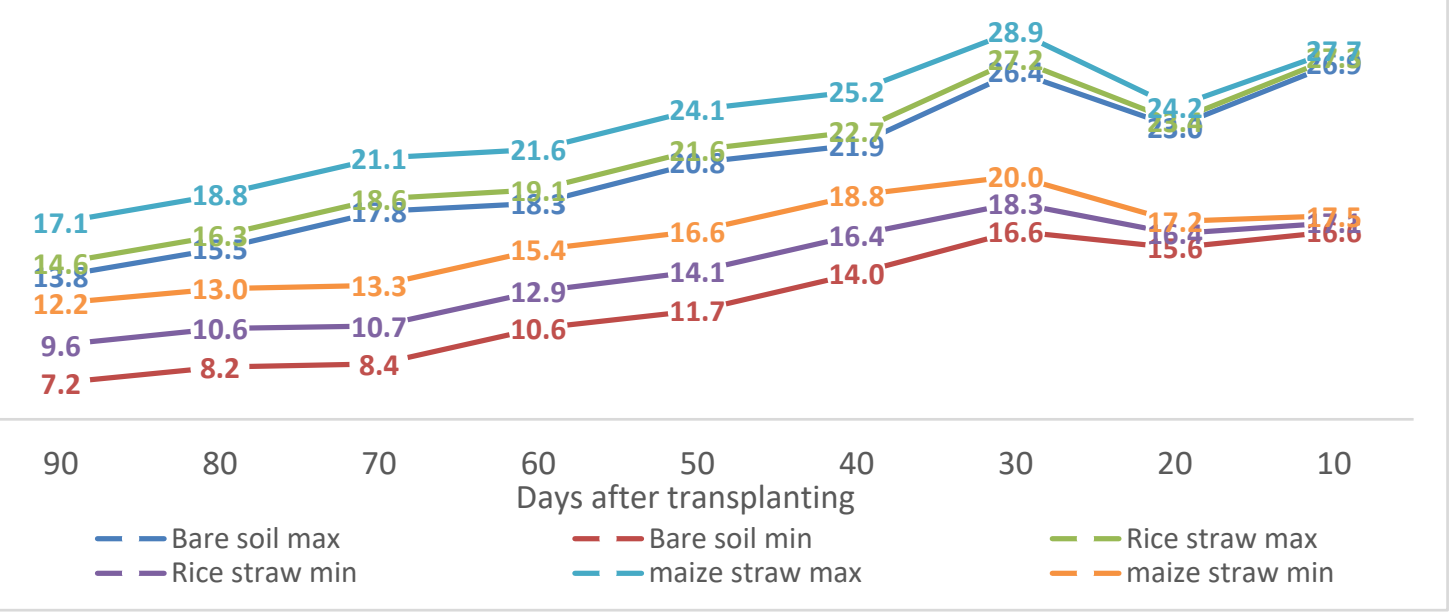

Figure 1 Average max. \& min. soil temperatures under two types of organic mulch (rice straw \& maize straw) during season of 2019

Average max. \& min. soil temperatures $\left(\mathrm{c}^{\circ}\right)$ of bare soil and under two types of organic mulch (rice straw \& maize straw) during season of 2020

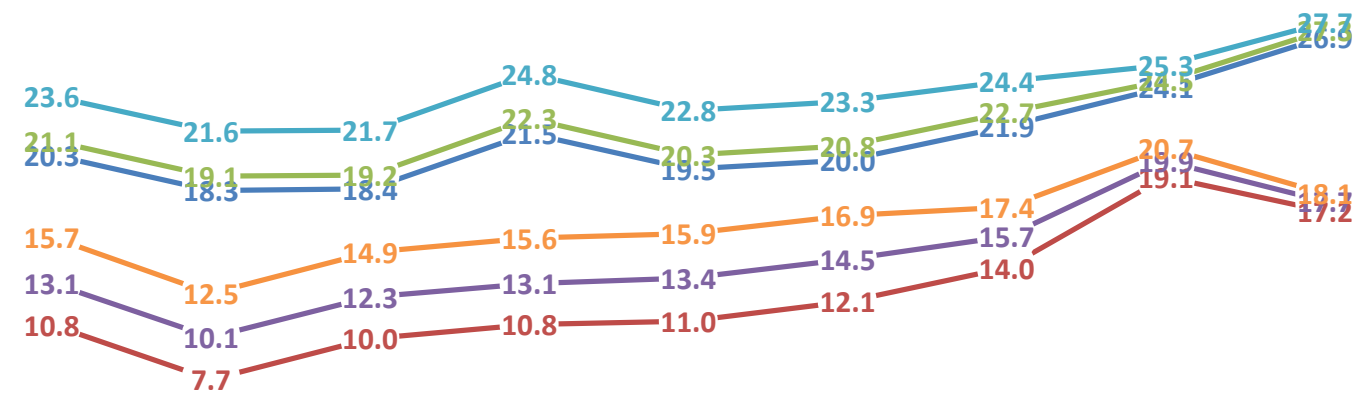

$90 \quad 80 \quad 70$

$60 \quad 50 \quad 40$

Days after transplanting

- Bare soil max
- - Rice straw min

- Bare soil min
- maize straw max

30

20

10

- - Rice straw max

- maize straw min

Figure 2 Average max. \& min. soil temperature under two types of organic mulch (rice straw \& maize straw) during season of 2020

The highest recorded average maximum soil temperature was observed in plots covered with maize straw followed by those cover with rice straw. Whereas, maize straw mulch increased the average maximum soil temperature by 2.5 to $3.3^{\circ} \mathrm{C}$ above average bare soil temperature. However, rice straw mulch increased average maximum soil temperature about $1{ }^{\circ} \mathrm{C}$ above average bare soil temperature. The same range of increase was observed in both studied seasons.

Similarly, application of maize straw soil mulch increased average minimum soil temperature by 3.5 to $5{ }^{\circ} \mathrm{C}$ above average minimum temperature of bare soil. Moreover, rice straw mulching increased average minimum soil temperature by 1.5 to $2.5^{\circ} \mathrm{C}$ above average minimum temperature of bare soil. The same range of increase was observed in both studied seasons. Obtained result was in harmony with Horton et al., [26]. They indicated that organic mulch has a special mechanism to modify soil temperature during winter season by reducing heat loss from soil providing thermal insulation layer. Moreover, organic mulch reflects the short waves (Albedo effect) more than soil. Both thermal insulation and albedo effect reduce soil temperature capacity and this resulted in less soil temperature extremes under organic mulch. In addition, Zhao [27] reported that, mulches change soil temperature by about 0.5 to $3.0{ }^{\circ} \mathrm{C}$ depending on many factors. 


\subsection{Vegetative growth}

\subsubsection{Plant height}

Data presented in Table (1) showed the positive effect of applying organic mulches, LEDs light colors and their interaction on plant height of green bean plants. In general, application of organic mulch leads to significant increase in plant height compared to control. The highest values of plant height of green bean plants were obtained with maize straw treatment $(41.34 \& 43.16 \mathrm{~cm})$ followed by rice straw treatment $(37.88 \& 40.37 \mathrm{~cm})$, while the lowest value of plant height was recorded with the control treatment $(33.37 \& 35.07 \mathrm{~cm})$ during 2019 and 2020, respectively.

Concerning effect of LEDs light colors, the greatest value of plant height obtained with blue LED light color treatment (44.40 \& $46.34 \mathrm{~cm})$, followed by "red + blue" LEDs light colors treatment $(39.72 \& 42.10 \mathrm{~cm})$, then by control treatment $(34.89 \& 36.50 \mathrm{~cm})$. While, red LED light color treatment gave the lowest value of plant height $(31.11 \& 33.19 \mathrm{~cm})$, during 2019 and 2020, respectively.

Considering the interaction between tested factors, interaction between "maize straw and blue LED light color" increased plant height of green bean plants more than other treatments (48.86 \& $50.12 \mathrm{~cm}$ during 2019 and 2020 , respectively). Contrary, interaction between "control (bare soil) and red LED light color" reduced plant height to record 27.26 \& $29.07 \mathrm{~cm}$ during 2019 and 2020, respectively.

Table 1 Effect of organic mulch types, LEDs light colors and their interaction on plant height (cm) for green bean plants during $2019 \& 2020$ seasons

\begin{tabular}{|l|l|l|l|l|}
\hline \multirow{2}{*}{ LEDs light Colors } & \multicolumn{4}{|c|}{ Organic mulch } \\
\cline { 2 - 5 } & Maize straw & Rice straw & Control & Mean \\
\cline { 2 - 5 } & First season & $27.26 \mathrm{f}$ & $31.11 \mathrm{D}$ \\
\hline \multirow{2}{*}{ Red } & $34.56 \mathrm{~d}$ & $31.5 \mathrm{e}$ & $39.79 \mathrm{c}$ & $44.40 \mathrm{~A}$ \\
\hline Blue & $48.86 \mathrm{a}$ & $44.55 \mathrm{~b}$ & $35.75 \mathrm{~d}$ & $39.72 \mathrm{~B}$ \\
\hline Red + Blue & $43.44 \mathrm{~b}$ & $39.96 \mathrm{c}$ & $34.89 \mathrm{C}$ \\
\hline Control & $38.49 \mathrm{c}$ & $35.52 \mathrm{~d}$ & $30.66 \mathrm{e}$ & 3 \\
\hline Mean & $41.34 \mathrm{~A}$ & $37.88 \mathrm{~B}$ & $33.37 \mathrm{C}$ & \\
\hline \multirow{2}{*}{ Red } & Second season & & \\
\hline Blue & $36.70 \mathrm{~d}$ & $33.80 \mathrm{e}$ & $29.07 \mathrm{f}$ & $33.19 \mathrm{D}$ \\
\hline Red + Blue & $50.12 \mathrm{a}$ & $47.02 \mathrm{~b}$ & $41.88 \mathrm{c}$ & $46.34 \mathrm{~A}$ \\
\hline Control & $46.19 \mathrm{~b}$ & $42.75 \mathrm{c}$ & $37.35 \mathrm{~d}$ & $42.10 \mathrm{~B}$ \\
\hline Mean & $39.63 \mathrm{c}$ & $37.89 \mathrm{~d}$ & $31.97 \mathrm{e}$ & $36.50 \mathrm{C}$ \\
\hline
\end{tabular}

\subsubsection{Number of leaves}

As shown in Table (2), number of leaves/plant was affected significantly by application of organic mulch types. The highest number of leaves noticed with maize straw treatment (26.06 \& 28.40 leaves/plant), followed by rice straw treatment (24.34 \& 26.3 leaves/plant), while, the lowest number of leave numbers recoded with the control treatment (21.06 \& 22.75 leaves/plant), during 2019 and 2020, respectively.

Regarding effect of LEDs light colors, it positively increased studied characteristic. The "red + blue" LEDs light gave highest value among other treatments (28.92 \& 31.29 leaves/plant, during 2019 and 2020, respectively). While, blue LED light, the control (without LED light) and the red LED light treatments ranked second, third and fourth, respectively.

Regarding interaction between organic mulch and LEDs light colors, application of "red+ blue" LEDs light interacted with maize straw increased green bean number of leaves significantly compared with other treatments $(31.50 \& 34.68$ 
leaves/plant, during 2019 and 2020, respectively). While, interaction between control (bare soil) treatment + red LED light obtained the lowest value of leave numbers (17.60 \& 18.97 leaves/plant, during 2019 and 2020; respectively).

Table 2 Effect of organic mulch types, LEDs light colors and their interaction on number of leaves/plant for green bean plants during $2019 \& 2020$ seasons

\begin{tabular}{|l|l|l|l|l|}
\hline \multirow{2}{*}{ LEDs light Colors } & \multicolumn{4}{l}{ Organic mulch } \\
\cline { 2 - 5 } & Maize straw & Rice straw & Control & Mean \\
\cline { 2 - 5 } & First season \\
\hline \multirow{2}{*}{ Red } & $20.65 \mathrm{f}$ & $19.45 \mathrm{f}$ & $17.60 \mathrm{~h}$ & $19.23 \mathrm{D}$ \\
\hline Blue & $27.34 \mathrm{c}$ & $25.75 \mathrm{~d}$ & $21.94 \mathrm{ef}$ & $25.01 \mathrm{~B}$ \\
\hline Red + Blue & $31.50 \mathrm{a}$ & $29.82 \mathrm{~b}$ & $25.45 \mathrm{~d}$ & $28.92 \mathrm{~A}$ \\
\hline Control & $24.76 \mathrm{~d}$ & $22.35 \mathrm{e}$ & $19.25 \mathrm{~g}$ & $22.12 \mathrm{C}$ \\
\hline Mean & $26.06 \mathrm{~A}$ & $24.34 \mathrm{~B}$ & $21.06 \mathrm{C}$ & \\
\hline \multirow{2}{*}{ Red } & Second season & & \\
\hline Blue & $22.24 \mathrm{e}$ & $21.19 \mathrm{f}$ & $18.97 \mathrm{~g}$ & $20.80 \mathrm{D}$ \\
\hline Red + Blue & $30.15 \mathrm{~b}$ & $27.25 \mathrm{c}$ & $23.41 \mathrm{de}$ & $26.94 \mathrm{~B}$ \\
\hline Control & $34.68 \mathrm{a}$ & $31.29 \mathrm{~b}$ & $27.90 \mathrm{c}$ & $31.29 \mathrm{~A}$ \\
\hline Mean & $26.54 \mathrm{c}$ & $24.37 \mathrm{~d}$ & $20.71 \mathrm{f}$ & $23.87 \mathrm{C}$ \\
\hline \multirow{2}{*}{$*$ Alphabetic inside the table refers to analysis of variance of statistically assess the data. }
\end{tabular}

\subsubsection{Steam diameter}

Data illustrated in Table (3) showed that green bean plants cultivated with applied maize straw, as organic mulch, increased the steam diameter by $0.62 \& 0.64 \mathrm{~cm}$, followed by rice straw treatment by $0.60 \& 0.61 \mathrm{~cm}$, and control treatment by $0.59 \& 0.60 \mathrm{~cm}$, during 2019 and 2020 ; respectively.

It was noticed that green bean plants grown under red LED light recorded greatest steam diameter $(0.70 \& 0.71 \mathrm{~cm}$, during 2019 and 2020; respectively). While, blue LED light obtained the lowest values $(0.49 \& 0.50 \mathrm{~cm}$, during 2019 and 2020; respectively).

Focusing on interaction, "maize straw + red LED light" gave the highest steam diameter values of green bean plants among other treatments ( $0.72 \& 0.74 \mathrm{~cm}$, during 2019 and 2020; respectively). Whereas, interaction between "blue LED light \& without mulch (control)" recorded the lowest values of steam diameter $(0.47 \& 0.48 \mathrm{~cm}$, during 2019 and 2020; respectively).

\subsubsection{Number of branches/plant}

Data in Table (4) indicated that, amount of branches/plant of green bean affected significantly by organic mulch, LEDs light colors and their interaction. The highest number of branches obtained with maize straw $(8.0 \& 8.19 \mathrm{branch} / \mathrm{plant}$, during 2019 and 2020; respectively). Furthermore, blue LED light treatment increased number of branches/plant (8.35 \& 8.52 branch/plant, during 2019 and 2020; respectively) followed by "red+ blue" LEDs light (7.52 \& 7.69 branch/plant, during 2019 and 2020; respectively), red LED light color (6.58 \& 6.73 branch/plant, during 2019 and 2020; respectively) and without LED light (as control) treatments (5.69 \& 5.83 branch/plant, during 2019 and 2020; respectively), which, placed the second, third and fourth places, respectively.

Highlighting effect of interaction, the highest number of branches observed interaction between "blue LED light \& maize straw treatment" ( $9.32 \& 9.55$ branch/plant, during 2019 and 2020; respectively), while, the lowest values indicated by interaction between "control (without mulch) \& control (without LED light)" (4.58 \& 4.72 branch/plant, during 2019 and 2020; respectively). 
Table 3 Effect of organic mulch types, LEDs light colors and their interaction on steam diameter $(\mathrm{cm})$ for green bean plants during 2019 \& 2020 seasons

\begin{tabular}{|l|l|l|l|l|}
\hline \multirow{2}{*}{ LED light Colors } & \multicolumn{4}{l}{ Organic mulch } \\
\cline { 2 - 5 } & Maize straw & \multicolumn{4}{l|}{ Rice straw } & Control & Mean \\
\cline { 2 - 5 } & \multicolumn{4}{|l|}{ First season } \\
\hline Red & $0.72 \mathrm{a}$ & $0.70 \mathrm{~b}$ & $0.68 \mathrm{c}$ & $0.70 \mathrm{~A}$ \\
\hline Blue & $0.50 \mathrm{~g}$ & $0.49 \mathrm{~g}$ & $0.47 \mathrm{~h}$ & $0.49 \mathrm{D}$ \\
\hline Red + Blue & $0.67 \mathrm{c}$ & $0.65 \mathrm{~d}$ & $0.64 \mathrm{~d}$ & $0.65 \mathrm{~B}$ \\
\hline Control & $0.58 \mathrm{e}$ & $0.56 \mathrm{f}$ & $0.55 \mathrm{f}$ & $0.56 \mathrm{C}$ \\
\hline Mean & $0.62 \mathrm{~A}$ & $0.60 \mathrm{~B}$ & $0.59 \mathrm{C}$ & \\
\hline & Second season & & \\
\hline Red & $0.74 \mathrm{a}$ & $0.71 \mathrm{~b}$ & $0.69 \mathrm{c}$ & $0.71 \mathrm{~A}$ \\
\hline Blue & $0.51 \mathrm{~g}$ & $0.51 \mathrm{~g}$ & $0.48 \mathrm{~h}$ & $0.50 \mathrm{D}$ \\
\hline Red + Blue & $0.69 \mathrm{c}$ & $0.66 \mathrm{~d}$ & $0.66 \mathrm{~d}$ & $0.67 \mathrm{~B}$ \\
\hline Control & $0.60 \mathrm{e}$ & $0.57 \mathrm{f}$ & $0.56 \mathrm{f}$ & $0.58 \mathrm{C}$ \\
\hline Mean & $0.64 \mathrm{~A}$ & $0.61 \mathrm{~B}$ & $0.60 \mathrm{C}$ & \\
\hline
\end{tabular}

${ }^{*}$ Alphabetic inside the table refers to analysis of variance of statistically assess the data.

Table 4 Effect of organic mulch types, LEDs light colors and their interaction on number of branches/plant for green bean plants during $2019 \& 2020$ seasons

\begin{tabular}{|l|l|l|l|l|}
\hline \multirow{2}{*}{ LEDs light Colors } & \multicolumn{4}{l}{ Organic mulch } \\
\cline { 2 - 5 } & Maize straw & \multicolumn{4}{l|}{ Rice straw } & Control & Mean \\
\cline { 2 - 5 } & \multicolumn{4}{|l|}{ First season } \\
\hline Red & $7.60 \mathrm{~cd}$ & $6.45 \mathrm{f}$ & $5.68 \mathrm{~g}$ & $6.58 \mathrm{C}$ \\
\hline Blue & $9.32 \mathrm{a}$ & $8.20 \mathrm{~b}$ & $7.54 \mathrm{~d}$ & $8.35 \mathrm{~A}$ \\
\hline Red + Blue & $8.24 \mathrm{~b}$ & $7.75 \mathrm{c}$ & $6.58 \mathrm{f}$ & $7.52 \mathrm{~B}$ \\
\hline Control & $6.85 \mathrm{e}$ & $5.65 \mathrm{~g}$ & $4.58 \mathrm{~h}$ & $5.69 \mathrm{D}$ \\
\hline Mean & $8.00 \mathrm{~A}$ & $7.01 \mathrm{~B}$ & $6.10 \mathrm{C}$ & \\
\hline & Second season & & \\
\hline Red & $7.76 \mathrm{~cd}$ & $6.66 \mathrm{f}$ & $5.77 \mathrm{~g}$ & $6.73 \mathrm{C}$ \\
\hline Blue & $9.55 \mathrm{a}$ & $8.35 \mathrm{~b}$ & $7.67 \mathrm{~d}$ & $8.52 \mathrm{~A}$ \\
\hline Red + Blue & $8.45 \mathrm{~b}$ & $7.95 \mathrm{c}$ & $6.68 \mathrm{f}$ & $7.69 \mathrm{~B}$ \\
\hline Control & $7.01 \mathrm{e}$ & $5.75 \mathrm{~g}$ & $4.72 \mathrm{~h}$ & $5.83 \mathrm{D}$ \\
\hline Mean & $8.19 \mathrm{~A}$ & $7.18 \mathrm{~B}$ & $6.21 \mathrm{C}$ & \\
\hline
\end{tabular}

* Alphabetic inside the table refers to analysis of variance of statistically assess the data.

\subsubsection{Fresh weight of total plant}

Illustrated data in Table (5) indicated that, application maize straw treatment lead to increase total plant fresh weight of green bean plants (124.52 \& $126.50 \mathrm{~g} /$ plant, during 2019 and 2020; respectively) followed by rice straw treatment (117.15 \& $118.95 \mathrm{~g} /$ plant, during 2019 and 2020; respectively), which, placed second order. When, treatment without 
organic mulch (control) recorded lowest values of total plant fresh weight $(110.67 \& 112.73 \mathrm{~g} / \mathrm{plant}$, during 2019 and 2020; respectively).

Table 5 Effect of organic mulch types, LEDs light colors and their interaction on total plant fresh weight (g) for green bean plants during $2019 \& 2020$ seasons

\begin{tabular}{|c|c|c|c|c|}
\hline \multirow{3}{*}{ LEDs light Colors } & \multicolumn{4}{|c|}{ Organic mulch } \\
\hline & Maize straw & Rice straw & Control & Mean \\
\hline & \multicolumn{4}{|l|}{ First season } \\
\hline Red & 120.35 cde & $117.61 \mathrm{de}$ & $113.52 \mathrm{ef}$ & 117.16B \\
\hline Blue & 133.82ab & 128.94abc & $124.45 \mathrm{bcd}$ & $129.07 \mathrm{~A}$ \\
\hline Red + Blue & $138.67 \mathrm{a}$ & $134.78 \mathrm{a}$ & $131.56 \mathrm{ab}$ & $135.00 \mathrm{~A}$ \\
\hline Control & $105.24 \mathrm{f}$ & $87.28 \mathrm{~g}$ & $73.16 \mathrm{~h}$ & $88.56 \mathrm{C}$ \\
\hline \multirow[t]{2}{*}{ Mean } & $124.52 \mathrm{~A}$ & 117.15B & $110.67 \mathrm{C}$ & \\
\hline & \multicolumn{4}{|c|}{ Second season } \\
\hline Red & $122.46 \mathrm{c}$ & $118.85 d$ & $115.70 d$ & $119.00 \mathrm{~B}$ \\
\hline Blue & $135.75 a b$ & 130.79abc & $126.35 \mathrm{bcd}$ & $130.96 \mathrm{~A}$ \\
\hline Red + Blue & $140.41 \mathrm{a}$ & $136.61 \mathrm{ab}$ & 133.65ab & $136.89 \mathrm{~A}$ \\
\hline Control & $107.36 \mathrm{e}$ & $89.53 f$ & $75.22 \mathrm{~g}$ & $90.70 \mathrm{C}$ \\
\hline Mean & $126.50 \mathrm{~A}$ & 118.95B & $112.73 \mathrm{C}$ & \\
\hline
\end{tabular}

On other hand, applying LEDs light colors enhanced total plant fresh weight of green bean plants compared to control treatment (without LED light). The greatest value of total plant fresh weight of green bean plants observed with "red + blue" LEDs light treatment (40.06 \& $40.27 \mathrm{~g}$ /plant, during 2019 and 2020; respectively) Furthermore, control (without LED light) treatment gave the lowest values (88.56 \& $90.70 \mathrm{~g} /$ plant, during 2019 and 2020; respectively).

Regarding the interaction between organic mulch and LEDs light colors data in table (5) showed, that plants grown under "maize straw plus "red + blue" LED light treatment increased total plant fresh weight of green bean plants, when compared with other treatments (138.67 \& $140.41 \mathrm{~g}$ /plant, during 2019 and 2020; respectively). Whereas, interaction between "control (without mulch) + control (without LED light)" recorded lowest value of plant fresh weight (73.16 \& $75.22 \mathrm{~g} /$ plant, during 2019 and 2020; respectively).

\subsubsection{Dry weight of total plant}

Data in Table (6) indicated that, applying maize straw treatment increased total dry weight of green bean plants (29.31 \& $30.38 \mathrm{~g} /$ plant, during 2019 and 2020 ; respectively), followed by rice straw treatment $(25.73 \& 26.28 \mathrm{~g} / \mathrm{plant}$, during 2019 and 2020; respectively), which ranked second, while, treatment without organic mulch (control) gave the lowest values (21.95 \& $22.69 \mathrm{~g} /$ plant, during 2019 and 2020; respectively).

Regarding effect of LEDs light colors, the highest total dry weight of green bean plants obtained with "red + blue" LEDs light treatment ( $40.06 \& 40.27 \mathrm{~g} /$ plant, during 2019 and 2020; respectively), followed by the blue LED light color treatment (27.59 \& $28.63 \mathrm{~g} /$ plant, during 2019 and 2020; respectively), and the red LED light color treatment (19.61 \& $20.27 \mathrm{~g} /$ plant, during 2019 and 2020; respectively). Furthermore, control treatment (without LED light) gave the lowest values of $15.39 \& 16.03 \mathrm{~g} /$ plant, during 2019 and 2020, respectively.

Regarding interaction between organic mulch and LEDs light colors, data in Table (6) showed a significant increase in total dry weight of green bean plants grown under maize straw plus "red + blue" LEDs light treatment (44.74 \& 45.86 g/plant, during 2019 and 2020; respectively). While, interaction between "control (without mulch) + control (without LED light) treatment recorded the lowest values (12.15 \& $12.41 \mathrm{~g} /$ plant, during 2019 and 2020; respectively). 
Table 6 Effect of organic mulch types, LEDs light colors and their interaction on total plant dry weight (g) for green bean plants during $2019 \& 2020$ seasons

\begin{tabular}{|c|c|c|c|c|}
\hline \multirow{3}{*}{ LEDs light Colors } & \multicolumn{4}{|c|}{ Organic mulch } \\
\hline & Maize straw & Rice straw & Control & Mean \\
\hline & \multicolumn{4}{|l|}{ First season } \\
\hline Red & $21.66 \mathrm{fg}$ & $19.58 \mathrm{gh}$ & $17.59 \mathrm{~h}$ & $19.61 \mathrm{C}$ \\
\hline Blue & $32.37 d$ & $27.98 \mathrm{e}$ & $22.43 \mathrm{f}$ & $27.59 B$ \\
\hline Red + Blue & $44.74 \mathrm{a}$ & $39.81 b$ & $35.63 c$ & 40.06A \\
\hline Control & $18.45 \mathrm{~h}$ & $15.56 \mathrm{i}$ & $12.15 \mathrm{j}$ & 15.39D \\
\hline \multirow[t]{2}{*}{ Mean } & $29.31 \mathrm{~A}$ & 25.73B & $21.95 \mathrm{D}$ & \\
\hline & \multicolumn{4}{|c|}{ Second season } \\
\hline Red & $22.72 \mathrm{ef}$ & $20.46 \mathrm{fg}$ & $17.62 \mathrm{~g}$ & $20.27 \mathrm{C}$ \\
\hline Blue & $33.58 \mathrm{c}$ & $28.80 d$ & $23.50 \mathrm{e}$ & $28.63 \mathrm{~B}$ \\
\hline Red + Blue & $45.86 a$ & $39.52 b$ & $36.43 c$ & $40.60 \mathrm{~A}$ \\
\hline Control & $19.35 \mathrm{~g}$ & $16.32 \mathrm{~h}$ & $12.41 \mathrm{i}$ & 16.03D \\
\hline Mean & $30.38 \mathrm{~A}$ & $26.28 B$ & $22.69 \mathrm{C}$ & \\
\hline
\end{tabular}

*Alphabetic inside the table refers to analysis of variance of statistically assess the data.

\subsection{Chemical components of leaves}

\subsubsection{Chlorophyll reading}

Treatments means in Table (7) showed that organic mulch type treatments had highest chlorophyll reading in leaves compared to control (without mulch) treatment. Moreover, there is no significant difference between applying maize straw and rice straw on chlorophyll contents in green bean leaves.

On other hand, blue LED light color and "red + blue" LEDs colors treatments lead to increase chlorophyll reading in leaves of green bean without any significant difference between them (41.53 \& $41.80 \mathrm{~g} / \mathrm{plant}$ and 40.77 \& $40.96 \mathrm{~g} / \mathrm{plant}$ for blue LED light color and "red + blue" LEDs light colors, during 2019 and 2020, respectively), followed by red LED light color treatment. While, control (without LED light) treatment decreased chlorophyll reading in leaves (36.55 \& $36.64 \mathrm{~g} /$ plant, during 2019 and 2020; respectively). Also, maize straw plus blue LED light color treatment recorded the highest chlorophyll reading in leaves, (42.45 \& $42.88 \mathrm{~g} /$ plant, during 2019 and 2020; respectively), while, without mulch (control) + without LED light (control) treatment reduced it (35.19 \& 35.28 g/plant, during 2019 and 2020 ; respectively).

\subsubsection{Nitrogen, phosphorus and potassium contents}

Results in Tables $(8,9$ and 10$)$ indicated that organic mulch treatments increased N, P and K \% contents compared to control (without mulch). Whereas, the highest values of $\mathrm{N}, \mathrm{P}$ and $\mathrm{K} \%$ contents in green bean leaves noticed with application of maize straw treatment $(2.82 \& 2.88 \%, 0.52 \& 0.53 \%$ and $2.71 \& 2.72 \%$ for N, P and K; during 2019 and 2020 , respectively). Followed by rice straw ( $2.72 \& 2.77 \%, 0.49 \& 0.51 \%$ and $2.64 \& 2.65 \%$ for N, P and K; respectively during 2019 and 2020) and control (without mulch) (2.57 \& $2.63 \%, 0.45 \& 0.46 \%$ and $2.56 \& 2.57 \%$ for N, P and K; during 2019 and 2020, respectively). Except for P contents, where no significant difference found between applying maize straw treatment or rice straw treatment.

Regarding LEDs light colors found that, "red + blue" LED light treatment recorded the greatest values of $\mathrm{N}, \mathrm{P}$ and $\mathrm{K} \%$ contents in leaves of green bean $(2.84 \& 2.89 \%, 0.56 \& 0.57 \%$ and $2.80 \& 2.81 \%$ for N, P and K; respectively during 2019 and 2020), followed by blue LED light color and red LED light color treatments, respectively. When, the lowest of $\mathrm{N}, \mathrm{P}$ and $\mathrm{K} \%$ contents in leaves observe with without LED light treatment (as control) (2.55 \& $2.61 \%, 0.41 \& 0.42 \%$ and 2.43 \& $2.45 \%$ for N, P and K; during 2019 and 2020, respectively). 
Table 7 Effect of organic mulch types, LEDs light colors and their interaction on chlorophyll reading of green bean plants during 2019 \& 2020 seasons

\begin{tabular}{|c|c|c|c|c|}
\hline \multirow{3}{*}{ LEDs light Colors } & \multicolumn{4}{|c|}{ Organic mulch } \\
\hline & Maize straw & Rice straw & Control & Mean \\
\hline & \multicolumn{4}{|l|}{ First season } \\
\hline Red & $39.82 \mathrm{c}$ & $38.53 d$ & $36.12 \mathrm{fg}$ & $38.16 \mathrm{~B}$ \\
\hline Blue & $42.45 \mathrm{a}$ & 41.62ab & $40.52 b c$ & $41.53 \mathrm{~A}$ \\
\hline Red + Blue & $41.55 \mathrm{ab}$ & $40.89 \mathrm{bc}$ & $39.86 c$ & $40.77 \mathrm{~A}$ \\
\hline Control & 37.69de & $36.76 \mathrm{ef}$ & $35.19 \mathrm{~g}$ & $36.55 \mathrm{C}$ \\
\hline \multirow[t]{2}{*}{ Mean } & $40.38 \mathrm{~A}$ & $39.45 \mathrm{~A}$ & $37.92 \mathrm{~B}$ & \\
\hline & \multicolumn{4}{|c|}{ Second season } \\
\hline Red & $39.98 d$ & $38.68 \mathrm{e}$ & $36.32 \mathrm{gh}$ & 38.33B \\
\hline Blue & $42.88 a$ & 41.78abc & $40.75 \mathrm{~cd}$ & $41.80 \mathrm{~A}$ \\
\hline Red + Blue & 41.95ab & $40.98 \mathrm{bcd}$ & $39.96 \mathrm{~d}$ & $40.96 \mathrm{~A}$ \\
\hline Control & $37.77 \mathrm{ef}$ & $36.88 \mathrm{fg}$ & $35.28 \mathrm{~h}$ & $36.64 \mathrm{C}$ \\
\hline Mean & $40.65 \mathrm{~A}$ & $39.58 \mathrm{~A}$ & $38.08 \mathrm{~B}$ & \\
\hline
\end{tabular}

${ }^{*}$ Alphabetic inside the table refers to analysis of variance of statistically assess the data.

Table 8 Effect of organic mulch types, LEDs light colors and their interaction on nitrogen (\%) in green bean plants during $2019 \& 2020$ seasons

\begin{tabular}{|l|l|l|l|l|}
\hline \multirow{2}{*}{ LEDs light Colors } & \multicolumn{4}{|l|}{ Organic mulch } \\
\cline { 2 - 5 } & Maize straw & \multicolumn{4}{l|}{ Rice straw } & \multicolumn{2}{l|}{ Control } & Mean \\
\cline { 2 - 5 } & First season \\
\hline Red & $2.78 \mathrm{c}$ & $2.67 \mathrm{~d}$ & $2.52 \mathrm{f}$ & $2.66 \mathrm{C}$ \\
\hline Blue & $2.89 \mathrm{a}$ & $2.78 \mathrm{c}$ & $2.61 \mathrm{e}$ & $2.76 \mathrm{~B}$ \\
\hline Red + Blue & $2.91 \mathrm{a}$ & $2.87 \mathrm{~b}$ & $2.73 \mathrm{c}$ & $2.84 \mathrm{~A}$ \\
\hline Control & $2.69 \mathrm{~d}$ & $2.55 \mathrm{f}$ & $2.42 \mathrm{~g}$ & $2.55 \mathrm{D}$ \\
\hline Mean & $2.82 \mathrm{~A}$ & $2.72 \mathrm{~B}$ & $2.57 \mathrm{C}$ & \\
\hline & Second season & \multicolumn{3}{|l}{} \\
\hline Red & $2.83 \mathrm{c}$ & $2.72 \mathrm{de}$ & $2.58 \mathrm{f}$ & $2.71 \mathrm{C}$ \\
\hline Blue & $2.95 \mathrm{ab}$ & $2.83 \mathrm{c}$ & $2.68 \mathrm{e}$ & $2.82 \mathrm{~B}$ \\
\hline Red + Blue & $2.98 \mathrm{a}$ & $2.91 \mathrm{~b}$ & $2.79 \mathrm{c}$ & $2.89 \mathrm{~A}$ \\
\hline Control & $2.74 \mathrm{~d}$ & $2.61 \mathrm{f}$ & $2.47 \mathrm{~g}$ & $2.61 \mathrm{D}$ \\
\hline Mean & $2.88 \mathrm{~A}$ & $2.77 \mathrm{~B}$ & $2.63 \mathrm{C}$ & \\
\hline
\end{tabular}

*Alphabetic inside the table refers to analysis of variance of statistically assess the data. 
Table 9 Effect of application organic mulch types, LEDs light colors and their interaction on phosphorus (\%) contents in green bean plants during $2019 \& 2020$ seasons

\begin{tabular}{|l|l|l|l|l|}
\hline \multirow{2}{*}{ LEDs light Colors } & \multicolumn{3}{|l|}{ Organic mulch } \\
\cline { 2 - 5 } & Maize straw & Rice straw & Control & Mean \\
\cline { 2 - 5 } & First season \\
\hline Red & $0.49 \mathrm{de}$ & $0.47 \mathrm{ef}$ & $0.44 \mathrm{fg}$ & $0.47 \mathrm{C}$ \\
\hline Blue & $0.55 \mathrm{bc}$ & $0.51 \mathrm{~d}$ & $0.47 \mathrm{ef}$ & $0.51 \mathrm{~B}$ \\
\hline Red + Blue & $0.59 \mathrm{a}$ & $0.56 \mathrm{ab}$ & $0.52 \mathrm{~cd}$ & $0.56 \mathrm{~A}$ \\
\hline Control & $0.44 \mathrm{fg}$ & $0.41 \mathrm{~g}$ & $0.37 \mathrm{~h}$ & $0.41 \mathrm{D}$ \\
\hline Mean & $0.52 \mathrm{~A}$ & $0.49 \mathrm{~A}$ & $0.45 \mathrm{~B}$ & \\
\hline & Second season & \\
\hline Red & $0.50 \mathrm{ef}$ & $0.48 \mathrm{fg}$ & $0.44 \mathrm{~g}$ & $0.47 \mathrm{C}$ \\
\hline Blue & $0.56 \mathrm{abc}$ & $0.53 \mathrm{ce}$ & $0.48 \mathrm{fg}$ & $0.52 \mathrm{~B}$ \\
\hline Red + Blue & $0.60 \mathrm{a}$ & $0.58 \mathrm{ab}$ & $0.54 \mathrm{bce}$ & $0.57 \mathrm{~A}$ \\
\hline Control & $0.45 \mathrm{~g}$ & $0.44 \mathrm{~g}$ & $0.38 \mathrm{~h}$ & $0.42 \mathrm{D}$ \\
\hline Mean & $0.53 \mathrm{~A}$ & $0.51 \mathrm{~A}$ & $0.46 \mathrm{~B}$ & \\
\hline
\end{tabular}

Table 10 Effect of organic mulch types, LEDs light colors and their interaction on potassium (\%) contents in green bean plants during $2019 \& 2020$ seasons

\begin{tabular}{|l|l|l|l|l|}
\hline \multirow{2}{*}{ LEDs light Colors } & \multicolumn{4}{|l|}{ Organic mulch } \\
\cline { 2 - 5 } & Maize straw & \multicolumn{4}{l|}{ Rice straw } & Control & Mean \\
\cline { 2 - 5 } & First season \\
\hline Red & $2.63 \mathrm{f}$ & $2.59 \mathrm{~g}$ & $2.51 \mathrm{i}$ & $2.58 \mathrm{C}$ \\
\hline Blue & $2.78 \mathrm{c}$ & $2.72 \mathrm{~d}$ & $2.66 \mathrm{e}$ & $2.72 \mathrm{~B}$ \\
\hline Red + Blue & $2.86 \mathrm{a}$ & $2.80 \mathrm{~b}$ & $2.73 \mathrm{~d}$ & $2.80 \mathrm{~A}$ \\
\hline Control & $2.55 \mathrm{~h}$ & $2.43 \mathrm{j}$ & $2.32 \mathrm{k}$ & $2.43 \mathrm{C}$ \\
\hline Mean & $2.71 \mathrm{~A}$ & $2.64 \mathrm{~B}$ & $2.56 \mathrm{C}$ & \\
\hline & Second season & \multicolumn{3}{|l|}{} \\
\hline Red & $2.65 \mathrm{~d}$ & $2.60 \mathrm{e}$ & $2.52 \mathrm{f}$ & $2.59 \mathrm{C}$ \\
\hline Blue & $2.80 \mathrm{~b}$ & $2.75 \mathrm{c}$ & $2.67 \mathrm{~d}$ & $2.74 \mathrm{~B}$ \\
\hline Red + Blue & $2.87 \mathrm{a}$ & $2.82 \mathrm{~b}$ & $2.75 \mathrm{c}$ & $2.81 \mathrm{~A}$ \\
\hline Control & $2.57 \mathrm{e}$ & $2.44 \mathrm{~g}$ & $2.33 \mathrm{~h}$ & $2.45 \mathrm{D}$ \\
\hline Mean & $2.72 \mathrm{~A}$ & $2.65 \mathrm{~B}$ & $2.57 \mathrm{C}$ & \\
\hline
\end{tabular}

As for the effect of their interaction noticed that application of maize straw plus "red + blue" LEDs light colors treatment increased green bean leaves contents from N, P and K more than other treatments $(2.91 \& 2.98 \%, 0.59 \& 0.60 \%$ and $2.86 \& 2.87 \%$ for N, P and K; during 2019 and 2020, respectively). Whereas, without mulch (control) + without LED 
light (control) reduced their contents $(2.42 \& 2.47 \%, 0.37 \& 0.38 \%$ and $2.32 \& 2.33 \%$ for N, P and K; during 2019 and 2020, respectively).

\subsection{Yield and its components}

\subsubsection{Number of pods/plant}

Data in Table (11) showed that the two types of organic mulch treatment increased number of pods per plant, without any significant difference between them, compared to control treatment (without mulch).

On the other hand, the blue LED light color and the "red + blue" LEDs light treatment recoded the greatest number of pods per plant, without any significant difference between them (46.57 \& 48.61 pods/plant and $45.75 \& 47.73$ pods/plant, during 2019 and 2020, respectively). The red LED light treatment ranked the third. Whereas, the control treatment (without LED light) decreased the number of pods/plant (36.87 \& 36.43 pods/plant; respectively during 2019 and 2020).

Table (11) also showed that, the maize straw + blue LED light color treatment gave the highest number of pods/plant compared to other treatments (48.65 \& 50.50 pods/plant, respectively, during 2019 and 2020). While, the control treatment (without mulch + without LED light) obtained the lowest number (33.27 \& 34.87 pods/plant; respectively, during 2019 and 2020).

Table 11 Effect organic mulch types, LEDs light colors and their interaction on the number of pods/plant of green bean during 2019 \& 2020 seasons

\begin{tabular}{|c|c|c|c|c|}
\hline \multirow{3}{*}{ LEDs light Colors } & \multicolumn{4}{|c|}{ Organic mulch } \\
\hline & Maize straw & Rice straw & Control & Mean \\
\hline & \multicolumn{4}{|l|}{ First season } \\
\hline Red & $42.25 \mathrm{~cd}$ & 40.68de & $38.52 \mathrm{fg}$ & $40.48 B$ \\
\hline Blue & $48.65 a$ & 46.78ab & $44.29 c$ & 46.57A \\
\hline Red + Blue & 47.98ab & $45.89 b$ & $43.35 c$ & $45.74 \mathrm{~A}$ \\
\hline Control & 39.79ef & $37.54 \mathrm{~g}$ & $33.27 \mathrm{~h}$ & $36.87 \mathrm{C}$ \\
\hline \multirow[t]{2}{*}{ Mean } & $44.67 \mathrm{~A}$ & $42.72 \mathrm{~A}$ & 39.86B & \\
\hline & \multicolumn{4}{|c|}{ Second season } \\
\hline Red & $44.86 \mathrm{~d}$ & $42.53 \mathrm{e}$ & $40.11 f$ & $42.50 \mathrm{~B}$ \\
\hline Blue & $50.50 \mathrm{a}$ & 48.87ab & $46.45 \mathrm{~cd}$ & $48.61 \mathrm{~A}$ \\
\hline Red + Blue & $49.96 \mathrm{ab}$ & $47.89 \mathrm{bc}$ & $45.34 d$ & $47.73 \mathrm{~A}$ \\
\hline Control & $41.14 \mathrm{ef}$ & $39.27 f$ & $34.87 \mathrm{~g}$ & $38.43 \mathrm{C}$ \\
\hline Mean & $46.62 \mathrm{~A}$ & $44.64 \mathrm{~A}$ & 41.69B & \\
\hline
\end{tabular}

\subsubsection{Average pod weight}

Result in Table (12) indicated that maize treatment lead to the increase of average pod weight of green bean plants compared to other treatments (3.68 \& $3.77 \mathrm{~g} /$ pod; respectively, during 2019 and 2020). While, control treatment (without mulch) reduced average pod weight (3.41\&3.52 g/pod; respectively, during 2019 and 2020).

Concerning LEDs light colors effect, data in Table (12) indicated that "red + blue" LEDs light treatment produced green pods with greater values of average pod weight, followed by blue LED light color and red LED light color treatments (3.99 \& $4.10 \mathrm{~g} /$ pod, $3.67 \& 3.76 \mathrm{~g} /$ pod and $3.47 \& 3.57 \mathrm{~g} / \mathrm{pod}$, respectively, during 2019 and 2020). Whereas, plants grown without LED light (control) gave the lowest values (3.02 \& 3.14 g/pod; respectively, during 2019 and 2020 ). 
The interaction between organic mulch types and LEDs light colors showed that, "red + blue" LEDs light interacted with maize straw treatment had higher average pod weight than other treatments $(4.12$ \& $4.26 \mathrm{~g} /$ pod; respectively during 2019 and 2020). While, lowest values of this parameter were obtained with the control (bare soil without LED light) treatment (2.93 \& $3.07 \mathrm{~g} /$ pod; respectively, during 2019 and 2020).

\subsubsection{Fiber content in pods}

Table (13) showed the role of organic mulch types, LEDs light colors and their interaction on fiber contents in green bean pods. In general, fiber contents in pods decreased significantly by applying both types of tested organic mulch compared to control treatment (without mulching).

Therefore, plants grown with maize straw treatment produced the lowest value of fiber contents, followed by rice straw treatment (8.44 \& $8.47 \%$ and $8.73 \& 8.78 \%$, respectively, during 2019 and 2020), while, plants grown without mulch (control) treatment increased pod fiber contents (9.01 \& $9.04 \%$, respectively, during 2019 and 2020).

The "red + blue" LEDs light treatment produced the lowest value of fiber contents in green bean pods (7.39 \& $7.42 \%$; respectively, during 2019 and 2020). While, plants cultivated without LED light treatment (control) gave the highest value of fiber contents in pods (10.25 \& $10.28 \%$, respectively, during 2019 and 2020).

Positive role of interaction was obtained through the results illustrated in Table (13). Applying maize straw together with "red + blue" LEDs light lead to decrease green bean pod contents of fiber $(7.11$ \& $7.14 \%$; respectively during 2019 and 2020). On the other hand, control treatment (without mulch and without LED light control) increased this parameter (10.80 \& $10.83 \%$, respectively, during 2019 and 2020.

Table 12 Effect of organic mulch types, LEDs light colors and their interaction on average pod weight (g) of green bean plants during $2019 \& 2020$ seasons

\begin{tabular}{|c|c|c|c|c|}
\hline \multirow{3}{*}{ LEDs light Colors } & \multicolumn{4}{|c|}{ Organic mulch } \\
\hline & Maize straw & Rice straw & Control & Mean \\
\hline & \multicolumn{4}{|l|}{ First season } \\
\hline Red & $3.64 d$ & $3.45 \mathrm{e}$ & $3.33 \mathrm{f}$ & $3.47 \mathrm{C}$ \\
\hline Blue & $3.84 \mathrm{c}$ & $3.66 \mathrm{~d}$ & $3.52 \mathrm{e}$ & $3.67 \mathrm{~B}$ \\
\hline Red + Blue & $4.12 \mathrm{a}$ & $3.97 \mathrm{~b}$ & $3.87 \mathrm{c}$ & $3.99 \mathrm{~A}$ \\
\hline Control & $3.11 \mathrm{~g}$ & $3.01 \mathrm{~h}$ & $2.93 \mathrm{i}$ & $3.02 \mathrm{D}$ \\
\hline \multirow[t]{2}{*}{ Mean } & $3.68 \mathrm{~A}$ & $3.52 \mathrm{~B}$ & $3.41 \mathrm{C}$ & \\
\hline & \multicolumn{4}{|c|}{ Second season } \\
\hline Red & $3.75 \mathrm{e}$ & $3.56 \mathrm{f}$ & $3.37 \mathrm{~g}$ & $3.56 \mathrm{C}$ \\
\hline Blue & $3.85 \mathrm{~cd}$ & $3.78 \mathrm{de}$ & $3.65 f$ & $3.76 \mathrm{~B}$ \\
\hline Red + Blue & $4.26 \mathrm{a}$ & $4.07 \mathrm{~b}$ & $3.98 \mathrm{c}$ & $4.10 \mathrm{~A}$ \\
\hline Control & $3.23 \mathrm{~h}$ & $3.12 \mathrm{i}$ & $3.07 j$ & $3.14 \mathrm{D}$ \\
\hline Mean & $3.77 \mathrm{~A}$ & $3.63 \mathrm{~B}$ & $3.52 \mathrm{C}$ & \\
\hline
\end{tabular}

${ }^{*}$ Alphabetic inside the table refers to analysis of variance of statistically assess the data.

\subsubsection{Early yield per plant}

Data in Table (14) showed that, applying organic mulch lead to enhancing positively early yield per plant of green bean, compared to the control treatment (without mulch). The highest values of early yield/plant of green bean was noticed with maize straw treatment followed by rice straw treatment $(74.19 \& 75.28$ and 71.39 \& 72.52 (g) yield/plant, respectively, during 2019 and 2020.

Furthermore, blue LED light color and "red + blue" LEDs light treatments had a high early yield/plant of green bean pods (81.11 \& 82.22 and $79.69 \& 80.79$ yield/plant (g), respectively, during 2019 and 2020), without any significant 
difference between the two treatments. Followed by the red LED light and the control treatments $(71.39 \& 72.52$ and $53.84 \& 54.89$ yield/plant, during 2019 and 2020, respectively).

Table 13 Effect of organic mulch types, LEDs light colors application and their interaction on fiber contents (\%) in green bean pods during 2019 \& 2020 seasons

\begin{tabular}{|l|l|l|l|l|}
\hline \multirow{2}{*}{ LEDs light Colors } & \multicolumn{4}{l}{ Organic mulch } \\
\cline { 2 - 5 } & Maize straw & \multicolumn{2}{l|}{ Rice straw } & \multicolumn{2}{l|}{ Control } & Mean \\
\cline { 2 - 5 } & \multicolumn{4}{|l|}{ First season } \\
\hline \multirow{2}{*}{ Red } & $8.64 \mathrm{de}$ & $8.76 \mathrm{de}$ & $8.89 \mathrm{~d}$ & $8.76 \mathrm{~B}$ \\
\hline Blue & $8.25 \mathrm{f}$ & $8.53 \mathrm{ef}$ & $8.72 \mathrm{de}$ & $8.50 \mathrm{~B}$ \\
\hline Red + Blue & $7.11 \mathrm{~h}$ & $7.44 \mathrm{~g}$ & $7.63 \mathrm{~g}$ & $7.39 \mathrm{C}$ \\
\hline Control & $9.75 \mathrm{c}$ & $10.20 \mathrm{~b}$ & $10.80 \mathrm{a}$ & $10.25 \mathrm{~A}$ \\
\hline Mean & $8.44 \mathrm{~A}$ & $8.73 \mathrm{~B}$ & $9.01 \mathrm{C}$ & \\
\hline & Second season & & \\
\hline Red & $8.67 \mathrm{e}$ & $8.79 \mathrm{de}$ & $8.92 \mathrm{~d}$ & $8.79 \mathrm{~B}$ \\
\hline Blue & $8.28 \mathrm{f}$ & $8.53 \mathrm{ef}$ & $8.75 \mathrm{de}$ & $8.52 \mathrm{~B}$ \\
\hline Red + Blue & $7.14 \mathrm{~h}$ & $7.47 \mathrm{~g}$ & $7.66 \mathrm{~g}$ & $7.42 \mathrm{C}$ \\
\hline Control & $9.78 \mathrm{c}$ & $10.23 \mathrm{~b}$ & $10.83 \mathrm{a}$ & $10.28 \mathrm{~A}$ \\
\hline Mean & $8.47 \mathrm{~A}$ & $8.76 \mathrm{~B}$ & $9.04 \mathrm{C}$ & \\
\hline
\end{tabular}

${ }^{*}$ Alphabetic inside the table refers to analysis of variance of statistically assess the data.

Table 14 Effect of organic mulch types, LEDs light colors and their interaction on early yield/plant (g) in green bean plants during 2019 \& 2020 seasons

\begin{tabular}{|c|c|c|c|c|}
\hline \multirow{3}{*}{ LED light Color } & \multicolumn{4}{|c|}{ Organic mulch } \\
\hline & Maize straw & Rice straw & Control & Mean \\
\hline & \multicolumn{4}{|l|}{ First season } \\
\hline Red & $73.33 \mathrm{e}$ & $71.20 \mathrm{e}$ & $68.79 f$ & 71.11B \\
\hline Blue & $84.86 a$ & $80.55 b c$ & $77.93 \mathrm{~cd}$ & $81.11 \mathrm{~A}$ \\
\hline Red + Blue & $81.79 b$ & $79.82 \mathrm{bcd}$ & $77.45 d$ & 79.69A \\
\hline Control & $56.76 \mathrm{~g}$ & $53.97 \mathrm{~h}$ & $50.78 \mathrm{i}$ & $53.84 \mathrm{C}$ \\
\hline \multirow[t]{2}{*}{ Mean } & $74.19 \mathrm{~A}$ & 71.39B & $68.74 \mathrm{C}$ & \\
\hline & \multicolumn{4}{|c|}{ Second season } \\
\hline Red & $74.55 \mathrm{e}$ & $72.45 \mathrm{e}$ & $69.83 f$ & $72.28 \mathrm{~B}$ \\
\hline Blue & $85.95 a$ & $81.67 \mathrm{bc}$ & $79.03 \mathrm{~cd}$ & $82.22 \mathrm{~A}$ \\
\hline Red + Blue & $82.83 \mathrm{~b}$ & $80.86 \mathrm{bcd}$ & $78.67 d$ & $80.79 A$ \\
\hline Control & $57.80 \mathrm{~g}$ & $55.08 \mathrm{~h}$ & $51.79 \mathrm{i}$ & $54.89 \mathrm{C}$ \\
\hline Mean & $75.28 \mathrm{~A}$ & $72.52 B$ & 69.83C & \\
\hline
\end{tabular}

*Alphabetic inside the table refers to analysis of variance of statistically assess the data.

Regarding the interaction, cultivated green bean plants with maize straw + blue LED light treatment lead to increase early yield of pods per plant compared to other treatments (84.86 \& 85.95 yield/plant (g); respectively during 2019 and 
2020). Whereas, the control treatment (without mulch and without LED light) gave lower values of early yield/plant (50.78 \& 51.79 yield/plant (g), respectively, during 2019 and 2020).

\subsubsection{Total yield per plant}

Data in Table (15) indicated that total yield of green pods per plant of green bean was affected significantly by applying the tested factors during the two growing seasons. The highest total yield/plant of green bean was observed with maize straw treatment, followed by rice straw treatment (167.26 \& 177.09 and $152.78 \& 163.39$ yield/plant (g), during 2019 and 2020, respectively). While, the lowest value of total yield/plant of green pods was found with control (without mulch) treatment (143.51\& 148.05 yield/plant (g), during 2019 and 2020, respectively).

Moreover, "red + blue" LEDs light treatment produced heavy total yield from green pods per plant of green beans (187.31 \& 196.07 yield/plant (g), during 2019 and 2020, respectively). On the other hand, both blue LED light and red LED light colors were placed second and third places. Furthermore, treatment without LED light as (control) had low total yield from green pods/plant of common bean (113.74 \& 120.82 yield/plant (g), during 2019 and 2020 , respectively).

The interaction between organic mulch types and LEDs light colors had significant effect on total yield of common bean plants. The highest total yield of green pods/plant noticed with the interaction between maize straw and "red + blue" LEDs light treatment (197.68 \& 212.83 yield/plant (g), during 2019 and 2020, respectively). While, control treatment (without mulch and without LED light) gave the lowest total yield of green pods/plant (97.48 \& 107.05 yield/plant (g), during 2019 and 2020, respectively).

Table 15 Effect of organic mulch types, LEDs light colors and their interaction on total yield/plant (g) in green bean plants during 2019 \& 2020 seasons

\begin{tabular}{|c|c|c|c|c|}
\hline \multirow{3}{*}{ LEDs light Colors } & \multicolumn{4}{|c|}{ Organic mulch } \\
\hline & Maize straw & Rice straw & Control & Mean \\
\hline & \multicolumn{4}{|l|}{ First season } \\
\hline Red & $153.79 \mathrm{e}$ & $140.35 f$ & $132.27 \mathrm{~g}$ & 142.14C \\
\hline Blue & $186.82 \mathrm{~b}$ & $171.21 \mathrm{~cd}$ & $166.60 d$ & 174.88B \\
\hline Red + Blue & $197.68 \mathrm{a}$ & $186.58 \mathrm{~b}$ & $177.67 \mathrm{c}$ & $187.31 \mathrm{~A}$ \\
\hline Control & $130.75 \mathrm{~g}$ & $113.00 \mathrm{~h}$ & $97.48 \mathrm{i}$ & $113.74 \mathrm{D}$ \\
\hline \multirow[t]{2}{*}{ Mean } & $167.26 \mathrm{~A}$ & 152.78B & $143.51 \mathrm{C}$ & \\
\hline & \multicolumn{4}{|c|}{ Second season } \\
\hline Red & $168.23 d$ & $151.41 \mathrm{e}$ & $135.17 f$ & 151.60C \\
\hline Blue & $194.43 b$ & $184.73 c$ & $169.54 \mathrm{~d}$ & 182.90B \\
\hline Red + Blue & $212.83 a$ & $194.91 b$ & $180.45 c$ & $196.07 \mathrm{~A}$ \\
\hline Control & $132.88 f$ & $122.52 \mathrm{~g}$ & $107.05 \mathrm{~h}$ & $120.82 \mathrm{D}$ \\
\hline Mean & $177.09 \mathrm{~A}$ & 163.39B & $148.05 \mathrm{C}$ & \\
\hline
\end{tabular}

\section{Discussion}

Obtained results indicated the superiority of both organic mulch treatments (rice straw \& maize straw), compared with bare soil, concerning the enhancement of all measured characteristics related to plant growth (plant length, number of leaves per plant, stem diameter, number of branches/plant and fresh and dry weights), nutrient uptake (percentage of $\mathrm{N}, \mathrm{P}$ and $\mathrm{K}$ content) and crop yield (number of pods, average pod weight, early and total yield per plant). 
The above-mentioned trend of results was confirmed by several authors [5, 7, 4 and 28]. These obtained enhancements were explained by Barche et al., [29] on bases of positive effect of the organic mulch that appear in three different dimensions (physical, chemical and biological).

Physical effect of organic mulch due to its ability to change soil structure in the root zone area, which is reflected in increasing the growth of roots and nutrient uptake. In addition, organic mulch improves the soil aeration as well as water holding capacity $[29,28)$. Moreover, process of organic mulch decomposition presents a reach source of soil granulation throughout activation sticky substances secretion by micro-organisms.

Chemical effect of organic mulch presented through controlling $\mathrm{pH}$ of the soil to acidity or alkalinity. Mentioned effect concerning soil pH is mainly depending on kind of applied organic mulch. Most of organic mulch kinds slightly increase the $\mathrm{pH}$ [29]. Moreover, the organic mulch play a good role in adds a little amount of nutrient elements during process of decomposition.

Biological role of organic mulch became from its role as food for beneficial microorganism. As well as, stimulation microorganism's community through adding extra quantities of organic matter to the soil [30]. On the other hand, organic mulch modifies soil temperature to be constant which guarantee the continuous activity of beneficial microorganisms and new roots activity [31, 26, 8 and 7].

Focusing on the results related to the effect of tested LED light colors, it's noticeable that mixing both red and blue LED lights presents a superiority in large number of studied characteristics i.e. number of leaves, plant fresh weight, plant dry weight, N (\%), P(\%), K (\%), number of pods, early yield and total crop yield. Such excellence explained by both of [32] and [33] by the ability of both LED lights together (red and blue) to activate large number of photoreceptors compared to the effect of individual colors. Whereas, blue LED light affects cryptochromes, phytotropins and Flavinbinding Kelch (FKF1) that are initiated and involved in biosynthesis of hormones and secondary metabolites, stomatal activity and cell wall elasticity [34]. In addition, it leads to earliness in bud outgrowth, internode elongation, flowering and protection against biotic and abiotic factors. However, red LED light has the highest RPE, about $90 \%$, meaning the plants under red light gained more energy than the others [35].

Concerning effect of the solo blue LED light, it was found that the blue LED light increased both of plant height and chlorophyll reading (SPAD) compared to other tested LED light colors. Briggs and Christie, [36] reported that the blue color wavelength is between $400-520 \mathrm{~nm}$ where, Chlorophyll pigments obtain peak energy absorption at these wavelengths and strongly influence vegetative growth and development. More reports related to effect of blue light were presents by [32, 37, 38, 39, 40 and 41]. They indicated the ability of blue light to affect both of primary and secondary metabolism, volatile production, carotenoid and chlorophyll pigment bioaccumulation and intermodal length.

Similarly, red and far-red (700-800 nm) wavelengths are able to activate other photoreceptors (phytochromes) involved in the regulation of different processes through the plant life cycle (including seed germination, shade avoidance, flowering time, vegetative development, root growth and nutrient uptake) as reviewed by Demotes-Mainard S et.al [42].

\section{Conclusion}

Covering bare soil by organic mulch led to modified soil temperature and increased tested characteristics of common bean plants, when compared with bare soil, as open field condition. The greatest values of most studied parameters observed with chopped maize straw followed by chopped rice straw. Applying LEDs light colors had positive role on enhancing vegetative growth parameters, uptake nitration elements, pods characteristics and produced heavy early and total green pods more than open field conditions. Furthermore, the most favorable effect of LEDs light colors ranked as "red + blue", "blue color" and "red color", respectively, for most of the tested parameters. Combination between organic mulch types and LEDs light colors achieved highest results for most parameters, especially, chopped maize straw plus "red + blue" LEDs light treatment.

\section{Compliance with ethical standards}

\section{Disclosure of conflict of interest}

No conflict of interest. 


\section{References}

[1] Ndegwa AM, MN Muchui, SM Wachiuri, JN Kimamira. Evaluation of snap bean varieties for adaptability and pod quality. In: Proceedings of the 10th KARI Biennial Conference. Nairobi, Kenya. $13^{\text {th }}-17^{\text {th }}$ Nov. 2006.

[2] Borresen EC, N Jenkins-Puccetti, K Schmitz, DG Brown, A Pollack, A Fairbanks, M Wdowik, S Rao, TL Nelson, G Luckasen. A pilot randomized controlled clinical trial to assess tolerance and efficacy of navy bean and rice bran supplementation for lowering cholesterol in children. Glob. Pediatr. Health. 2017; 4: 1-10.

[3] Sadek II, AY Mohamed. Influence of colored net covering for low tunnels on microclimate, soil properties, growth, yield and quality of common bean plants (Phaseolus vulgaris L.). Scientific Journal of Recent Agricultural Science. 2019; 2(1): 1-28.

[4] Kader MA, M Senge, MA Mojid, K Ito. Soil \& Tillage Research Recent advances in mulching materials and methods for modifying soil environment. Soil \& Tillage Research. 2017; 168: 155-166.

[5] Kwambe XM, MT Masarirambi, PK Wahome, TO Oseni. The effects of organic and inorganic mulches on growth and yield of green bean (Phaseolus vulgaris L.) in a semi-arid environment. Agric. Biol. J. N. Am. 2015; 6(3): 8189.

[6] Alharbi A. Effect of mulch on soil properties under organic farming. 2017; 2(1961): 230-236.

[7] Moursy FS, FA Mostafa, NY Solieman. Polyethylene and rice straw as soil mulching: reflection of soil mulch type on soil temperature, soil borne diseases, plant growth and yield of tomato. Glo. J. Adv. Res. 2015; 2: 1496-1519.

[8] Chalker-Scott L. Impact of mulches on landscape plants and the environment - a review. J. Environ. Hortic. 2007; 25: 239-249.

[9] Rathore AL, AR Pal, KK Sahu. Tillage and mulching effects on water use, root growth and yield of rainfed mustard and chickpea grown after lowland rice. J. Sci. Food Agr. 1988; 78: 149-161.

[10] Abd El-Wahed MH, GA Baker, MM Ali, FA Abd El- Fattah. Evaluating the effects of drip deficit irrigation and thickness of rice straw mulch layer on beans crop and water use efficiency. Misr J. Ag. Eng. $2017 ; 34$ (1): 273 290.

[11] Nadalini S, P Zucchi, C Andreotti. Effects of blue and red LED lights on soilless cultivated strawberry growth performances and fruit quality. Eur. J. Hortic. Sci. 2017; 82: 12-20.

[12] Folta K, S Carvalho. Photoreceptors and control of horticultural plant traits. HortScience. 2015; 50: 1274-1280.

[13] Kopsell DA, CE Sams, TC Barickman, RC Morrow. Sprouting broccoli accumulate higher concentrations of nutritionally important metabolites under narrow-band light-emitting diode lighting J. Amer. Soc. Hort. Sci. 2014; 139: 469-477.

[14] Hammock HA, Kopsell DA, Sams CE. Narrowband Blue and Red LED Supplements Impact Key Flavor Volatiles in Hydroponically Grown Basil Across Growing Seasons. Front. Plant Sci. 2021; 1-15.

[15] Gangadhar BH, RK Mishra, G Pandian, SW Park. Comparative study of color, pungency, and biochemical composition in chili pepper (Capsicum annuum L.) under different light-emitting diode treatments. Hort Science. 2012; 47: 1729-1735.

[16] Son KH, MM Oh. Leaf shape, growth, and antioxidant phenolic compounds of two lettuce cultivars grown under various combinations of blue and red light-emitting diodes. HortScience. 2013; 48: 988-995.

[17] Piovene C, F Orsini, S Bosi, R Sanoubar, V Bregola, G Dinelli, G Gianquinto. Optimal red:blue ratio in led lighting for nutraceutical indoor horticulture. Sci. Hortic. 2015; 193: 202-208.

[18] Johkan MK, F Shoji, S Goto, SS Hashida, T Yoshihara. Blue light-emitting diode light irradiation of seedlings improves seedling quality and growth after transplanting in red leaf lettuce. HortScience. 2010; 45: 1809-1814.

[19] Mitchell C, Both AJ, Bourget M, Burr J, Kubota C, Lopez R, Morrow R, Runkle E. LEDs: The future of greenhouse lighting! Hortic. Sci. Focus. 2012; 52: 1-9.

[20] Pocock T. Light-emitting diodes and the modulation of specialty crops: Light sensing and signaling networks in plants. HortScience. 2015; 50: 1281-1284.

[21] VERCON. Common bean production. Bulletin No. 2007; 1085. 
[22] Chapman HD, Pratt PF. Methods of Analysis for Soils, Plants and Waters. Priced Publication 4034. University of California-Berkeley, Division of Agricultural Science. 1961.

[23] FAO (Food and Agriculture Organization). Soil and Plant Analysis. Soils Bulletin. 1980; 38(2): 250.

[24] AOAC. Official Methods of Analysis of the Association of Official Analytical Chemists, 18th ed., Association of Official Analytical Chemists, Arlington, Virginia, USA. 2005.

[25] SAS Institute. The SAS system for Microsoft Windows. Release 9. 1. SAS Inst., Cary, NC. 2005.

[26] Horton R, KL Bristow, GJ Kluitenberg, TJ Sauer. Crop residue effects on surface radiation and energy balance review. Theor. Appl. Climatol. 1996; 54(1-2): 27-37.

[27] Zhao H, YC Xiong, FM Li, RY Wang, SC Qiang, TF Yao, F Mo. Plastic film mulch for half growing-season maximized WUE and yield of potato via moisture-temperature improvement in a semi-arid agroecosystem. Agric. Water Manage. 2012; 104: 68-78.

[28] Sadek II, A. Y Mohamed NY, Solieman M Alyafei. Response of soil properties, growth, yield and fruit quality of cantaloupe Plants (Cucumis melo L.) to organic mulch. Merit Research Journal of Agricultural Science and Soil Sciences. 2019; 7(9): 106-122.

[29] Barche S, R Nair, PK Jain. A review of mulching on vegetable crops production. Eco. Env. \& Cons. 2015; 21(2): 859-866.

[30] Olsen JK, RK Gounder. Alternatives to polyethylene mulch film field assessment of transported materials in capsicum (Capsicum annuum L.). Austral. J. Expt. Agr. 2001; 41(1): 93-103.

[31] Martin PJ, R Poultney. Survival and growth of clove seedlings in Zanzibar. Effects of mulching and shade crops. J. Tropic. Agric. 1992; 69: 365-373.

[32] Christie JM. Phototropin blue-light receptors. Annu. Rev. Plant Biol. 2007; 58: 21-45.

[33] Galvao VC, C Fankhauser. Sensing the light environment in plants: photoreceptors and early signaling steps. Curr. Opin. Neurobiol. 2015; 34: 46-53.

[34] Huché-Thélier L, L Crespel, J Le Gourrierec, P Morel, S Sakr, N Leduc. Light signaling and plant responses to blue and UV radiations-Perspectives for applications in horticulture. Environ. Exp. Bot. 2016; 121: 22-38.

[35] Taiz L, E Zeiger, IM Møller, A Murphy. Plant Physiology and Development, 6th Edn. Sinauer Associates Inc., Sunderland, MA. 2014.

[36] Briggs WR, Christie JM. Phototropins 1 and 2: Versatile plant blue-light receptors. Trends Plant Sci. 2002; 7: 204210.

[37] Abney KR, DA Kopsell, CE Sams, S Zivanovic, DE Kopsell. UV-B radiation impacts shoot tissue pigment composition in Allium fistulosum L. cultigens. Sci. World J. 2013; 513867.

[38] Kopsell DA, CE Sams. Increases in shoot tissue pigments, glucosinolates, and mineral elements in sprouting broccoli after exposure to short-duration blue light from light emitting diodes. J. Am. Soc. Hortic. Sci. 2013; 138: 31-37.

[39] Metallo RM, DA Kopsell, CE Sams, NR Bumgarner. Influence of blue/red vs. white LED light treatments on biomass, shoot morphology, and quality parameters of hydroponically grown kale. Sci. Hortic. 2018; 235: 189197.

[40] Bantis F, A Koukounaras, AS Siomos, MN Fotelli, D Kintzonidis. Bichromatic red and blue LEDs during healing enhance the vegetative growth and quality of grafted watermelon seedlings. Sci. Hortic. 2020; 261: 109000.

[41] Samuoliene G, A Virsile, P Haimi, J Miliauskiene. Photoresponse to different lighting strategies during red leaf lettuce growth. J. Photochem. Photobiol. B. 2020; 202: 111726.

[42] Demotes-Mainard S, T Péron, A Corot. Plant responses to red and far-red lights, applications in horti-culture. Environmental and Experimental Botany. 2016; 121: 4-21. 